\title{
DISCURSOS CIUDADANOS ACERCA DE LA MUERTE EN CONDICIONES DE DIGNIDAD Y SOBRE LA POTENCIAL APLICACIÓN DE MEDIDAS DE ACOMPAÑAMIENTO EN ESTE PROCESO (REGIÓN METROPOLITANA, CHILE)
}

\begin{abstract}
Gonzalo Tassara $^{1}$, Catalina Valenzuela ${ }^{1}$, Luis Aravena ${ }^{1}$, Úrsula Sánchez ${ }^{2}$, Hernán Sandoval ${ }^{2}$
Resumen: El presente artículo expone los resultados de una investigación empírica que se ha adentrado en las experiencias y discursos ciudadanos respecto del tema de la muerte en dignidad y de la legitimidad de la implementación de medidas de acompañamiento de este proceso. A través de la realización y análisis de siete grupos de discusión, llevados a cabo en la Región Metropolitana de Santiago de Chile, se ha podido concluir la existencia de altos niveles de consenso entre la ciudadanía, respecto de la legitimidad de la implementación de medidas externas para terminar con la vida de pacientes que lo soliciten debido al padecimiento de enfermedades terminales, y de la necesidad de contar con una normativa legal que respalde su aplicación. Todo esto en el marco de una amplia valoración ciudadana de la autonomía personal y desde la narración de experiencias que revelan los grandes costos emocionales y monetarios que implica el cuidado de personas con enfermedades terminales.
\end{abstract}

Palabras clave: eutanasia, envejecimiento, opinión pública, políticas de la salud

Citizen speeches about death in conditions of dignity and about the potential application of accompanying measures in this process (Metropolitan Region, Chile)

\begin{abstract}
This article presents the results of empirical research that investigates the citizen's perspectives regarding the issue of death with dignity and the legitimacy of the implementation of measures to accompany this process. Through the implementation and analysis of seven discussion groups, carried out in the Metropolitan Region of Santiago de Chile, it can be concluded that there are high levels of consensus among the citizens supporting the need for implementation of external measures to end the life of patients who request it due to suffering related to terminal illnesses, and the need to have legal regulations that supports its application. All of this has been evaluated within the framework of a broad citizen assessment of personal autonomy and from the narration of experiences that reveal the great emotional and monetary burden involved in the process of caring for people with terminal illnesses.
\end{abstract}

Keywords: euthanasia, ageing, public opinion, health policy

Discursos cidadáos acerca da morte em condiçóes de dignidade e sobre a potencial aplicaçáo de medidas de acompanhamento neste processo (Regiáo Metropolitana, Chile)

Resumo: O presente artigo expõe os resultados de uma investigação empírica que adentrou as experiências e discursos cidadãos a respeito do tema da morte com dignidade e da legitimidade da implementaçáo de medidas de acompanhamento deste processo. Através da análise do obtido em sete grupos de discussão, levados a cabo na Regiáo Metropolitana de Santiago do Chile, se pode demonstrar a existência de altos níveis de consenso entre as pessoas no que diz respeito à legitimidade de implementar medidas externas para terminar com a vida de pacientes que as solicitem devido ao padecimento de enfermidades terminais e da necessidade de contar com uma normativa legal que respalde sua aplicaçáo. Tudo isto no contexto de uma ampla valorização cidadá da autonomia pessoal e a partir da narrativa de experiências que revelam os grandes custos emocionais e econômicos implicados no cuidado de pessoas com enfermidades terminais.

Palavras chave: eutanásia, envelhecimento, opinião pública, políticas de saúde

\footnotetext{
${ }^{1}$ Núcleo de Investigación Social Aplicada, Facultad de Ciencias Sociales, Universidad de Las Américas, UDLA, Chile. Gonzalo Tassara: https://orcid.org/0000-0002-3502-2459

Catalina Valenzuela: https://orcid.org/0000-0002-9019-6355

Luis Aravena: https://orcid.org/0000-0002-2837-5440

Correspondencia: gtassara@udla.cl

${ }^{2}$ Facultad de Ciencias de la Salud, Universidad de Las Américas, UDLA, Chile.

Úrsula Sánchez: https://orcid.org/0000-0002-4295-7084

Hernán Sandoval: https://orcid.org/0000-0002-3876-6357
} 


\section{Introducción}

En Chile, cada vez con mayor fuerza, se ha posicionado en el debate público el tema de la muerte en dignidad y de la implementación de medidas de acompañamiento para esta, cuestiones que cobran especial relevancia cuando se consideran las características demográficas actuales de la población chilena, las cuales evidencian un sostenido aumento de la proporción de adultos mayores $(1,2)$ y una significativa incidencia de enfermedades crónicas no transmisibles que desencadenan altos niveles de discapacidad y dependencia(3-5).

En este marco, aparece el debate en torno a las condiciones materiales y psicológicas necesarias para enfrentar enfermedades terminales, y acerca de las experiencias llevadas a cabo en otros países para hacer frente a los efectos del envejecimiento poblacional. Dentro de las distintas alternativas de abordaje de estas materias, hace algunas décadas aparece el tema de las condiciones que deben existir para garantizar la dignidad a la hora de morir y, en concreto, de algunas medidas para acompañar este proceso, como es el caso de la eutanasia, el suicidio asistido y la suspensión de la alimentación o del tratamiento médico aplicado a los pacientes.

\section{Discusión y avances legislativos en el mundo}

En el mundo, las primeras discusiones teóricas en el ámbito médico-filosófico respecto de la eutanasia se dan con fuerza a final de la década de los 80 y comienzos de los años 90 , principalmente en el hemisferio norte, siendo alimentadas, sobre todo, por algunos casos mediáticos de pacientes que solicitaban esta medida.

En este contexto se dan los primeros casos de legalización de la eutanasia, bajo criterios muy estrictos y solo en algunos estados de EE.UU. y en los países bajos (Holanda). Estos hechos llevaron a que se comenzara a discutir y reflexionar, globalmente, respecto de los procedimientos mismos y, sobre todo, sobre los límites éticos y filosóficos en la materia.

En el ámbito legislativo hay una serie de experiencias que aportan a esta discusión. Entre ellas se pueden destacar, por ejemplo, los casos de Ore- gón (EE.UU.), Holanda, Bélgica, Luxemburgo y, más cercano a nuestro contexto, Colombia, por contar con marcos normativos que permite la intervención externa en el proceso de muerte de pacientes. Con matices, la legislación de estos países establece como condiciones para su aplicación el carácter irreversible de la enfermedad que aqueja al paciente, encontrarse en una fase terminal de la misma, la existencia de sufrimiento físico o psicológico, la expresión de la voluntad del paciente de recibir el tratamiento letal y la importancia de la opinión médica, tanto en materia del juicio de la irreversibilidad del avance de la enfermedad, como para proporcionar la información médica necesaria al paciente, con el fin de que este pueda tomar la decisión en conocimiento de la gravedad real de su situación(5-8).

\section{La discusión acerca de la muerte en dignidad en Chile}

Esta discusión toma relevancia en Chile al comenzar el siglo XXI. En ella es posible identificar posiciones discursivas encontradas respecto del sentido y los procedimientos asociados a las medidas de acompańamiento en el proceso de muerte. Por una parte, encontramos posiciones que esgrimen la necesidad de estas prácticas basándose en los derechos de los pacientes, entre los que estarían incluidos la autonomía, la autodeterminación y la decisión sobre su propio cuerpo, así como el derecho genérico a morir con dignidad, el cual debiese ser respetado por los tribunales de justicia y el sistema médico(9). De la misma forma, en el marco de este debate, se señala la necesidad de no imponer creencias morales y religiosas u otras opciones culturales de un grupo a toda la sociedad, sobre todo considerando la desigualdad en el acceso de los pacientes a cuidados paliativos, generando que, en muchos casos, no tengan las condiciones suficientes para afrontar la enfermedad de una manera digna(10), y la legitimidad de la aplicación de ciertos tratamientos médicos como los cuidados paliativos, por ejemplo-que por causa colateral pudieran resultar en la muerte del paciente(11).

Por contrapartida, también se puede encontrar posiciones que, si bien comprenden lo complejo de la problemática de los pacientes terminales, muchas veces cronificados, consideran como 
infranqueables los límites impuestos por la ética respecto de los cuidados a otorgar a los pacientes. Desde estas posturas, a los pacientes se les debe acompañar utilizando los avances tecnológicos de la medicina actual, pero sin intervenir de manera externa en el proceso final de su vida, procurando la comprensión de la dignidad humana con base en el acompañamiento de sus últimos días, mediante la disposición de cuidados paliativos del sufrimiento(12), e incluso evitando algunos tratamientos, bajo la diferenciación entre matar o dejar morir, independiente de la disposición del paciente(13). En esta perspectiva, también podemos encontrar la lógica llamada de la "pendiente resbaladiza" (slippery slope), concepto desde el cual se argumenta que por la aprobación o flexibilización en determinadas materias - como por ejemplo la eutanasia bajo ciertas condiciones-, la sociedad terminará aceptando medidas que profundizan dichas situaciones hasta límites que sobrepasan el marco de lo aceptable éticamente, llevando por ejemplo a la aprobación de la eutanasia general en niños, pacientes sin discernimiento o con trastornos mentales $(8,14)$.

En materia legislativa, la eutanasia y el suicidio asistido se excluyen explícitamente de la Ley de Derechos y Deberes del Paciente, promulgada el año 2012. Si bien durante la década pasada se presentaron varias mociones tendientes a regular estas materias, hasta la fecha no se cuenta con un cuerpo legal al respecto, lo cual hace que toda ayuda a propiciar la muerte de pacientes terminales sea actualmente ilegal y penada por la ley(15).

\section{¿Y qué opina la ciudadanía?}

En el mundo, la mayor parte de la evidencia en esta materia proviene de estudios cuantitativos que han tenido como sujeto de estudio a la población europea. Por ejemplo, Cohen, Van Landeghem, Carpentier y Deliens(16) señalan, a partir del análisis de la encuesta European Values Study (EVS), que la aceptación de la eutanasia aumentó sostenidamente cada década entre la ciudadanía desde 1981 hasta 2008 en 11 de los 13 países de Europa occidental, cuestión que asocian a la secularización de la sociedad occidental y al concomitante aumento de la valoración que se hace de la autonomía personal.
Asimismo, Martínez y del Rosal(17) analizaron las actitudes de los ciudadanos españoles respecto de la regulación de la eutanasia y el suicidio asistido, encontrando que, para todos los casos planteados, los ciudadanos españoles apoyan mayoritariamente las practicas eutanásicas, variando el grado de acuerdo entre un 52,7\%, para el caso de la necesidad de regulación del suicidio médicamente asistido, hasta un 80,5\% para el caso de la aceptación de la práctica de la eutanasia en pacientes terminales y que padecen sufrimiento.

En contrapartida, la reseñada investigación de Cohen, Van Landeghem, Carpentier y Deliens(16) reporta niveles mayoritarios y consistentes de no aceptación de estas medidas en los países de Europa del Este, lo cual parece ser signo de sociedades ostensiblemente menos secularizadas que las de Europa Occidental. Complementariamente, Teodorescu, Sastre y Sorum(18) indican la existencia de niveles mayoritarios de no aceptación del suicidio asistido entre profesionales de la salud (85\%) y población "no experta" (51\%) en Rumania.

En el caso chileno, según la Encuesta $\operatorname{CEP(19),~}$ el $78 \%$ de la población opina que la eutanasia debería estar permitida en el país. De estos, un $22 \%$ seńala que eso debería ocurrir siempre que se solicitara y el 56\% que debería ser una medida aplicable en casos especiales. Estos datos revelan un aumento de siete puntos porcentuales en el nivel de aceptación general de la eutanasia respecto de la misma medición realizada en abril-mayo de 2017.

Más allá de lo reveladoras que puedan resultar estas cifras, podemos afirmar que aún existe una deuda en lo relativo al conocimiento respecto de las experiencias y opiniones que tienen los ciudadanos en esta materia, lo cual ha llevado a que sus miradas no hayan sido consideradas relevantes en el marco de la profusa discusión en el ámbito tanto académico como político-legislativo en Chile.

En este contexto, la investigación que ha dado origen a este artículo se puso como objetivo describir y analizar las experiencias y opiniones existentes entre los ciudadanos acerca de la temática del buen morir, intentando contestar a interrogantes tales como: ¿cuáles son las opiniones de la ciudadanía respecto de la muerte en dignidad? 
¿Cuáles son las experiencias de las personas respecto de la muerte de seres queridos y cuáles son las implicancias que estos procesos han tenido para sus vidas? ¿Qué ideas existen respecto de la legitimidad de las medidas asociadas al concepto de muerte en dignidad y con cuanta legitimidad cuentan? ¿Cuál es y debería ser el rol del Estado en estas materias? Todo esto con el fin de aportar elementos a la discusión de un modelo legal que regule la materia, desde la pertinencia y adecuación a las experiencias, necesidades y posiciones valóricas de la ciudadanía.

\section{Métodos}

El diseño de la presente investigación es descriptivo-explicativo y transversal, y ha sido realizada desde el enfoque cualitativo.

\section{Técnica de producción de información}

El dispositivo seleccionado ha sido el grupo de discusión, el cual puede ser entendido como una técnica de investigación de carácter cualitativo que, a través de la producción de una conversación socializada, intenta captar y analizar las representaciones simbólicas y los discursos ideológicos que emergen en la comunicación grupal(20).

A grandes rasgos, los temas tratados en los grupos de discusión fueron: conocimiento y valoración de las medidas de suspensión del tratamiento médico, suspensión de la alimentación, suicidio asistido, eutanasia activa y eutanasia pasiva, esto a partir de casos prácticos que sirvieron como gatilladores de la discusión; ideas y sentimientos generales respecto de la muerte; ideas y sentimientos que despiertan las ideas relativas a su propia muerte, en términos de su temporalidad ideal, condiciones en que debiese darse y consecuencias para las personas cercanas, y experiencias con la muerte y cuidado de personas cercanas en un contexto de enfermedad terminal.

\section{Participantes y muestra}

Se realizó un total de siete grupos de discusión, distribuidos geográficamente en cinco barrios y cuatro comunas de la ciudad de Santiago (Lo Prado, Huechuraba, Santiago Centro y La Florida), todas comunas donde habita mayoritariamente población de clase media y baja.

Los participantes de estos grupos fueron vecinos y vecinas de los barrios seleccionados. En cada uno de estos barrios se realizó una convocatoria abierta a quienes quisieran participar, siendo mayoritariamente mujeres adultas mayores quienes respondieron a este llamado, lo cual no parece extraño, tanto por su mayor cercanía con la muerte, como por ser quienes primordialmente realizan las labores de cuidado de personas enfermas terminales, lo cual hace que se muestren particularmente interesadas en entregar sus opiniones y experiencias en el tema. El rango de edad de los participantes fue desde los 23 a los 91 años. Sin embargo, se concentró en las de mayor edad: un $83 \%$ está sobre los 60 años, distribuyéndose un $43 \%$ entre los 71 y los 80 años, y un $15 \%$ por sobre esta edad. En términos de sexo, de los 64 participantes totales, 52 fueron mujeres $(81 \%)$ y 12 fueron hombres (19\%). En cada uno de los grupos de discusión participó un promedio de nueve personas.

Las actividades del trabajo en terreno fueron realizadas por los miembros del equipo de investigación, entre agosto y septiembre de 2019, y se llevaron a cabo en sedes vecinales, consejos de desarrollo local de salud y en dependencias de los centros de salud familiar de los barrios considerados.

\section{Procesamiento y análisis de la información}

El trabajo de cada uno de estos grupos de discusión fue grabado y transcrito, para posteriormente realizar un análisis de la información producida de acuerdo con la técnica del análisis temático(21), lo cual, en tanto método para el análisis de la información cualitativa, nos permitió identificar, organizar, analizar en profundidad y, finalmente, reportar patrones o temas relativos a la materia de estudio, a partir de una cuidadosa lectura de los textos recolectados, con el fin de inferir resultados que nos permitiesen una adecuada comprensión e interpretación de los fenómenos investigados.

\section{Temas éticos}

En el estudio se utilizó un consentimiento informado en el que se especificaba los objetivos de 
investigación, los alcances de su participación en ella, la confidencialidad, el derecho a retirarse y los datos del investigador principal en caso de dudas. Cada participante leyó y firmó este documento y obtuvo una copia firmada por el investigador presente en la actividad.

Además, la investigación cuenta con la aprobación del comité de ética de la universidad de la cual los autores son académicos.

\section{Resultados}

En esta sección, se presentan los principales resultados de los siete grupos de discusión que han constituido el trabajo de campo de la investigación. Luego del análisis realizado ha sido posible sistematizar la información producida identificando las temáticas más relevantes abordadas por la ciudadanía, las cuales se exponen a continuación.

\section{El poder del discurso biomédico}

Dentro de los contenidos más abordados en los grupos de discusión se puede identificar los relativos al rol de la medicina y de los médicos en la problemática de la dignidad al momento de morir. En este contexto, se reconoce el alto poder que tiene el modelo médico y los especialistas en el ámbito de la salud, a la hora de tomar la mayor parte de las decisiones referentes al tipo de tratamiento a aplicar a pacientes terminales y/o que padecen gran sufrimiento, sin ser tomada mayormente en consideración la opinión de las familias y de los propios pacientes. Así, existe relativo consenso entre los participantes del estudio en que las opiniones y la voluntad de los pacientes y sus familias debiesen ser consideradas a la hora de delinear el tratamiento médico en cada caso; lo cual, sin embargo, prácticamente no ocurre: prima una asimetría de poder en torno a la toma de decisiones, en la que el discurso médico prevalece la mayor parte de las veces.

"Estamos acostumbrados a que otras personas tengan que decidir por nosotros, no tenemos una vida donde podamos ejercer autónomamente nuestros derechos" (mujer 50 años, profesora, Huechuraba).

Se relatan diversos casos en que los mismos trata- mientos e intervenciones médicas muchas veces deterioran a los enfermos terminales, así como también se realizan intervenciones médicas que terminan extendiendo "artificialmente" la vida de los pacientes, lo que genera procesos muy dolorosos y desgastantes para las familias y el entorno de la persona enferma. En estas situaciones aparece con fuerza, desde los participantes en la investigación, la necesidad de incorporar la opinión y los deseos del paciente y sus familiares al mismo nivel que el diagnóstico biomédico, sin interferencia de posiciones personales/profesionales e incluso institucionales.

Respecto del rol profesional de los médicos, no existe total acuerdo. Por una parte, se aprecian posturas críticas respecto de su visión en torno a la muerte y los procedimientos implementados para extender la vida de los sujetos, las que en algunos casos se asocian con obtener beneficios económicos al tener pacientes hospitalizados por largos periodos de tiempo.

"Un sobrino que tuvo a su hijo durante cinco años con cáncer al cerebro, no lo podian operar. Se murió y le llegó la cuenta porque no todo era Auge. Dos millones y tanto y su hijo ya cumplió dos años de fallecido, imaginate estar pagando y su hijo ya no está" (mujer 57 años, dirigente vecinal, Huechuraba).

Pero, por otro lado, existen posiciones que relatan la necesidad de agotar los procedimientos médicos y tecnológicos para combatir las enfermedades y buscar tratamientos paliativos a enfermedades dolorosas, casos en los que se valora la función que pueda cumplir la medicina.

"Yo tengo el caso de la sobrina de una cuñada que tiene un cáncer grado 4, le dijeron no hay nada que hacer, hizo sus quimios, sus terapias, pero ya no hay nada que hacer" (mujer 71 años, pensionada, Lo Prado).

En la misma línea, tampoco existe completo acuerdo respecto de la aplicabilidad de la eutanasia a toda la población, sin realizar distinciones, puesto que existen posiciones que consideran que este procedimiento debiese estar orientado a personas mayores, con enfermedades terminales o que padezcan de gran sufrimiento físico. Sin embargo, otras posiciones sostienen que el proce- 
dimiento sí debiese ser aplicable a toda la población, sin distingos de edad o si el sufrimiento es físico o psíquico.

\section{Labores de cuidados de pacientes y el rol de gé- nero}

Respecto de los aspectos psicosociales de la problemática, aparece reiteradamente en las conversaciones la situación de las personas "cuidadoras" de familiares con enfermedades invalidantes, y la gran carga psíquica, emocional y económica que conlleva, así como la culpa asociada a la situación.

$\mathrm{Al}$ profundizar en esta situación, es claramente apreciable que en su mayoría son mujeres quienes se hacen cargo de las labores de cuidados, ya sea por razones económicas, emocionales o culturales. De esta manera, se identifica una sobrecarga de las tareas de cuidados de personas con enfermedades terminales en cierto grupo de la población, y hay acuerdo en que esta situación, si bien impacta a todo el grupo familiar, en su gestión se aprecia una responsabilidad preponderante de las mujeres, lo que se vivencia como una sobrecarga de funciones que los demás integrantes de la familia, sobre todo varones, no experimentan con la misma exigencia.

Estas experiencias parecen incidir en las opiniones y posturas de las mujeres participantes en el estudio, respecto de no llegar a ser una carga para sus familias en la eventualidad de llegar a padecer una situación de enfermedad invalidante, pues comprenden y han vivenciado la desigual distribución de responsabilidades y labores de cuidados dentro del sistema familiar, y los grandes costos que acarrea.

"Uno se enferma y enferma a los demás también... uno no quiere sufrir... no le tengo miedo a la muerte..." (mujer 67 años, dueña de casa, La Florida).

\section{Dilemas desde la espiritualidad y la moral}

Un factor que incide en los posicionamientos de las personas respecto de la problemática abordada tiene relación con aspectos culturales, como es la influencia de la religión cristiana y su influencia en los comportamientos de los sujetos. En los grupos de discusión se pudo observar que, en muchas ocasiones, las personas que se declaraban más practicantes del cristianismo mostraban en su discurso un alto grado de concordancia con los postulados religiosos respecto del valor y el respeto a la vida, en tanto don divino. Sin embargo, al situarlos en casos prácticos, estos juicios tendían a matizarse, dada la complejidad de llevarlos a cabo irrestrictamente en contextos de sufrimiento y dolor asociados al padecimiento de una enfermedad terminal, tanto para los directamente afectados como para sus familias.

"Es fuerte para la familia tener que aceptar. Igual es terrible, pero habría que aceptarla porque la persona ya no quiere más guerra, porque el cansancio, el agotamiento físico no da más, para tener a una persona sufriendo" (mujer 77 años, dueña de casa, La Florida).

Se aprecia que las familias creyentes experimentan una tensión respecto de ciertos postulados morales ligados a sus creencias religiosas, en los que la vida cobra un valor que está por sobre la decisión de los sujetos, situación que se ve confrontada a padecimientos extremos por parte de sus seres queridos. Esto las sitúa en un dilema ético que, sin embargo, y pese al dolor que pueda generar, en la mayor parte de los casos terminaría resolviéndose en evitar el padecimiento de la persona enferma.

"Yo tengo el caso de mi hermano, donde él quedó parapléjico. Piense que a él le paso este accidente cuando tenía 27 años. Ya lleva 60 años acostado en una cama de hospital y yo lo único... (llanto)... lo único que le pido a Dios es que se acuerde de él, porque solo está sufriendo y yo quiero mucho a mi hermano, pero me duele verlo asi" (hombre 84 años, pensionado, Santiago Centro).

En esta línea, también se aprecia otro factor que influye en los posicionamientos respecto del tema de estudio: las características de los casos en los cuales se podría aplicar la eutanasia. En general, se observa mayores dificultades para aceptar este tipo de procedimientos cuando son personas cercanas o con relaciones emocionales fuertes (padres, parejas, hijos); asimismo, cuando son niños o jóvenes, y cuando se trata de personas en las cuales la medicina aún cifra alguna posibilidad de recuperación. 


\section{Tensión en torno a la autonomía y la dependen- cia de los pacientes}

Es posible apreciar consenso entre los participantes respecto de la necesidad de tomar en consideración la decisión de la persona enferma, respetando así su autonomía y la dignidad en su última etapa de la vida. Como se ha mencionado, muchas veces dicha decisión es tomada por el equipo médico o familiares cercanos, coartando la libertad y la voluntad expresada en la decisión de los sujetos respecto de sus propias vidas y de las condiciones en las que quisieran enfrentar la muerte.

"En lo personal, yo también soy de la idea que yo le he dejado súper claro a mi familia (...) y les he dicho que si a mi me sucediera una cosa asi, yo no quisiera que me conectaran a nada" (mujer 55 años, trabajadora, Santiago Centro).

Al abordar estos temas en los grupos de discusión, se pudo observar los posicionamientos teóricos y filosóficos de los participantes respecto del sentido de la vida, a través del debate entre el valor intrínseco de la misma y la legitimidad de que otros humanos interfieran en su proceso natural; y, por otro lado, acerca de la necesidad de llegar a la última etapa de sus procesos vitales de manera digna, libre y ojalá alejada del sufrimiento y la carga emocional y económica que generan las enfermedades invalidantes, tanto para el paciente como para su entorno familiar.

"Yo creo que lo más razonable sería tomar una decisión en base a lo que esa persona quiera, ojalá fuera posible... para mí es lo que hay que respetar" (mujer 35 años, dirigente vecinal, Santiago Centro).

Respecto de este punto, los participantes le otorgan gran relevancia a la posibilidad de decidir por sí mismos las condiciones en que quieren morir, cuestión superior para quienes son adultos mayores, ya que consideran que se encuentran cerca de la etapa final de la vida y ven como una potencial amenaza no poder ejercer su autonomía.

"Yo creo que cuando uno está así, ya no hay nada que hacer, hay que aceptar la opinión del enfermo porque él es el que sufre los dolores" (hombre 76 años, pensionado, La Florida).
En este tema, se señala recurrentemente la necesidad de generar un mecanismo que permita preestablecer la voluntad personal, en el hipotético caso de padecer una enfermedad terminal y/o invalidante - tal como ocurre para el caso de la donación de órganos al morir-, que asegure el respeto a la autonomía de las personas, incluso en casos en los que, por las características de su padecimiento, las personas no puedan expresar su voluntad.

\section{El rol del Estado}

A partir del análisis realizado, podemos señalar la existencia de altos niveles de consenso respecto de que, en casos de enfermedades terminales o de gran sufrimiento, es comprensible la necesidad de contar con mecanismos legales para ayudar a morir de manera digna, por medio de acciones que vayan un paso más allá de los cuidados paliativos, y asumiendo que actualmente este tipo de prácticas se realizan en muchos casos, pero de forma encubierta, ya que son ilegales.

"En el caso de mi mamá, el médico le dijo: 'mire le voy a ir quitando el medicamento'... ella sufría mucho" (mujer 57 años, dirigente vecinal, Huechuraba).

En este sentido, se puede apreciar una diferencia sustantiva entre la que se concibe como la norma legal y la norma social. Si bien se reconoce que la norma legal en el país no contempla mecanismos jurídicos para dar término a la vida de manera externa, existe un amplio acuerdo respecto de que, en casos de dolor extremo o cuando es la propia persona enferma quién lo solicita, estas prácticas son legítimas y a la brevedad deberían estar permitidas por nuestro marco normativo.

"Es totalmente legitimo (el poner fin a la vida en los casos tratados), pero aun así no se firma la ley de la eutanasia" (mujer 76 años, dueña de casa, Santiago Centro).

"Todos estamos de acuerdo en que es legitimo quitarse la vida... Tiene que hacer lo que él quiera ¿qué más va a hacer? Se tiene que morir" (mujer 77 años, dueña de casa y cuidadora, Santiago Centro). 
Así, queda establecida la necesidad de que el Estado intervenga y tome postura respecto de esta problemática, considerando las complejas realidades que se viven en la sociedad, y no solo las posturas religiosas o morales de los representantes del sistema político, en tanto ellos no padecen la realidad de la mayor parte de la población. De esta manera, existe una interpelación directa al Estado en cuanto a regular esta problemática, ya que se estima que no se cuenta con una estructura normativa pertinente y que ponga en el centro el bienestar y la voluntad de la ciudadanía.

La situación de desregulación por parte del Estado, sumada a la desprotección frente a enfermedades terminales y de alto costo, impacta directamente en la población afectada. Según se nos relató, gran parte de las veces las familias deben enfrentan solas largos y costosos tratamientos médicos para combatir las enfermedades y sus padecimientos asociados, no contándose con los apoyos materiales ni emocionales suficientes para enfrentarlas. En otras palabras, se afirma que, si no existe la posibilidad de interrumpir la vida ante casos extremos, al menos debiese ser el Estado quien garantice el apoyo material y emocional a las familias, y no que sean estas quienes libren a su suerte la batalla contra el dolor y la enfermedad.

"En el caso en que uno estuviera solo, no tuviera familia, el Estado debería hacerse cargo... el Estado lo que más habla es del adulto mayor, pero no pasa nada pob" (hombre 80 años, carabinero retirado, Santiago Centro).

\section{Conclusiones y discusión}

Al comparar los resultados expuestos en esta investigación con los provenientes de estudios previos, podemos observar tendencias similares a lo que ha sido documentado para los países de Europa Occidental y para el propio caso chileno a través de sondeos cuantitativos(19), en relación con los altos niveles de aceptación de medidas tales como la eutanasia o el suicidio asistido. Los discursos que predominan en los grupos de discusión realizados muestran altos niveles de acuerdo con la legitimidad de la aplicación de este tipo de medidas, sobre todo en los casos de personas con enfermedades irreversibles y que causan gran sufrimiento, y con la necesidad de legislar en la materia.

Enmarcadas en este discurso de aceptación de la aplicación de medidas de acompañamiento para la muerte en dignidad, se pudo identificar dos vertientes discursivas predominantes: una proveniente de personas que, si bien estos temas les generan dilemas éticos, dada su mayor religiosidad o por tener una mirada más conservadora, la balanza, de todas formas, se termina inclinando hacia la aceptación de la aplicación de medidas de acompañamiento para la muerte en dignidad. Estas cuestiones parecen estar relacionadas con sus experiencias, y las de cercanos, en el cuidado de personas que han padecido enfermedades terminales, en las cuales han sido parte de procesos dolorosos y muy desgastantes, tanto emocional como económicamente. Desde estas posturas, el valor no negociable parece ser el de la dignidad al momento de morir, el cual no se estaría respetando en el actual estado de desregulación en la materia. En este sentido, la mayor parte de estas opiniones aluden a que se debe tratar de terminar con el sufrimiento de los seres queridos, otorgándoles la posibilidad de una muerte digna, por más complejo que sea adoptar esta decisión.

Adicionalmente, se pudo identificar una segunda vertiente discursiva, más secularizada y liberal desde lo valórico, desde la cual, además de ponerse el acento en la importancia de la dignidad al morir, se señala que debiese primar la voluntad de las personas enfermas por sobre la de los equipos médicos, las familias o el Estado, sobre todo en los casos en los que el sufrimiento experimentado sea muy grande. Todo esto en el marco de una alta valoración de la autonomía y la libertad de decisión personal, coherente con la secularización que ha vivido en la sociedad chilena durante las últimas décadas y la liberalización valórica que ha acompańado este proceso.

En esta sintonía, se puede observar una interpelación transversal al Estado, el cual, de acuerdo con los discursos levantados, pareciera estar en una posición éticamente paradójica: por una parte, no permite legalmente a las personas decidir si desean continuar con su vida en situaciones de enfermedades terminales y de gran sufrimiento; $y$, por otra, tampoco es el garante de que esa vida 
continúe en condiciones de dignidad, tanto de las personas enfermas como de sus familias, siendo ellas quienes deben asumir en mayor medida los costos emocionales y económicos de la situación. El llamado hacia el Estado entonces es que, tanto desde el ámbito legislativo como en materia de políticas públicas para el acompańamiento en el proceso de muerte de los enfermos terminales, se convierta en un garante del derecho de las personas a morir con dignidad.

Por último, considerando la señalada asimetría de poder en la toma de decisiones, en la que prácticamente siempre primaría la opinión de los equipos médicos por sobre la de los pacientes y sus familias, los resultados parecen mostrar la necesidad de abordar el proceso de muerte desde un enfoque centrado en los sujetos, atendiendo a la dimensión cultural y espiritual de la situación, más que en la tecnificación de procedimientos médicos. De esta manera, sería deseable una suerte de "humanización" del proceso médico en torno a la muerte, lo que coincide con lo postulado por diversos académicos respecto de la tensión existente entre comprender la muerte como un proceso natural o, bien, como un fracaso de las posibilidades técnicas de la medicina contemporánea $(9,10,12)$.

\section{Agradecimientos}

Queremos agradecer la colaboración, en calidad de ayudantes de investigación, de Bárbara Moscoso, Catalina Saavedra, Carlos Véliz y Luis Venegas, así como también al equipo de territorialistas de la Región Metropolitana, del Programa de Intervención Comunitaria de la Universidad de Las Américas, UDLA, cuyo trabajo fue fundamental para la difusión, convocatoria e implementación de las actividades en terreno. 
Discursos ciudadanos acerca de la muerte en condiciones de dignidad - Gonzalo Tassara et al.

\section{Referencias}

1. INE. Estimaciones y proyecciones de la población de chile 2002-2035 totales regionales, población urbana y rural. Chile: INE; 2019. Disponible en: https://www.ine.cl/docs/default-source/proyecciones-de-poblacion/publicaciones-yanuarios/base-2017/ine_estimaciones-y-proyecciones-2002-2035_base-2017_reg_\%C3\%A1rea_s\%C3\%ADntesis. pdf?sfvrsn=aaeb88e7_5

2. INE. Estimaciones y proyecciones de la población de Chile 1992-2050. Chile: INE; 2018. Disponible en: https://www.censo2017.cl/descargas/proyecciones/sintesis-estimaciones-y-proyecciones-de-la-poblacion-chile-1992-2050.pdf

3. Organización Panamericana de la Salud. Salud en las Américas. Panorama regional y perfiles de país. Washington: OPS; 2017. Disponible en: https:/www.paho.org/salud-en-las-americas-2017/wp-content/uploads/2017/09/Print-VersionSpanish.pdf.

4. Organización Panamericana de la Salud. Plataforma de información de salud para las Américas (Sistema de Consulta online). Plataforma disponible en: https://www.paho.org/data/index.php/es/ . Consulta realizada el 5/11/2019.

5. De Castro M, Antunes C, Marcon L, Andrade L, Rückl S, Andrade V. Eutanasia y suicidio asistido en países occidentales: una revisión sistemática. Revista Bioética 2016; 24(2): 355-367.

6. Biblioteca del Congreso Nacional. Aplicación de la Eutanasia: Bélgica, Colombia, Holanda y Luxemburgo. Chile: BCN; 2019. Disponible en: https://www.bcn.cl/obtienearchivo?id=repositorio/10221/27089/1/BCN_Eutanasia_Belgica_ Colombia_Holanda_Luxemburgo_FINAL.pdf.

7. Ministerio de Salud y Protección Social. Protocolo para la Aplicación del Procedimiento de Eutanasia en Colombia 2015. Colombia: Minsalud; 2015. Disponible en: https:/www.minsalud.gov.co/sites/rid/Lists/BibliotecaDigital/RIDE/DE/ CA/Protocolo-aplicacion-procedimiento-eutanasia-colombia.pdf.

8. De Miguel C, López A. Eutanasia y suicidio asistido: conceptos generales, situación legal en Europa, Oregón y Australia. Revista Medicina Paliativa 2006; 13(4): 207-215.

9. Zúñiga A. Derechos del paciente y eutanasia en Chile. Revista de Derecho (Valdivia) 2008; 21(2): 111-130.

10. Carrasco V, Crispi F. Eutanasia en Chile: una discusión pendiente. Revista Médica de Chile 2016; 144: 1598-1604.

11. Miranda A. Eutanasia, suicidio asistido y principio del doble efecto. Réplica al profesor Rodolfo Figueroa. Revista Médica de Chile 2012; 140(2): 261-265.

12. Taboada P. El derecho a morir con dignidad. Acta bioethica 2000; 6(1): 89-101.

13. Figueroa R. Matar, dejar morir y eutanasia en el proyecto de ley sobre derechos de las personas en la doctrina chilena. Revista Médica de Chile 2011; 139: 655-659.

14. Vega J. La práctica del suicidio asistido en Oregón y la "pendiente resbaladiza". Cuadernos de bioética 2007; 18(1): 55-70.

15. Biblioteca del Congreso Nacional. Eutanasia en la Legislación Chilena. Chile: BCN; 2019. Disponible en: https://obtienearchivo.bcn.cl/obtienearchivo?id=repositorio/10221/27133/1/BCN_eutanasia_en_la_legislacion_chilena_FINAL. pdf.

16. Cohen J, Van Landeghem P, Carpentier N, Deliens L. Different trends in euthanasia acceptance across Europe, 1981 2008. European Journal of Public Health 2013; 23(3): 378-380.

17. Martínez M, Del Rosal R. Regulación de la eutanasia y el suicidio asistido en España ¿¿Hacia qué modelo se dirige la opinión pública? Arbor: Ciencia, pensamiento y cultura 2014; 190(769): 794.

18. Teodorescu D, Sastre M, Sorum P. Romanian Lay People's and Health Professionals' Views about the Acceptability of Physician-Assisted Suicide. Universitas Psychologica 2019; 18(5): 1-7.

19. CEP. Estudio Nacional de Opinión Pública $N^{\circ}$ 82, Octubre-Noviembre 2018. Tema especial: Religión. Santiago de Chile: CEP; 2018. Disponible en: https:/www.cepchile.cl/cep/site/docs/20181218/20181218093906/encuestacep_oct_ nov2018_te_religion.pdf

20. Alonso L. E. La mirada cualitativa en sociología. Madrid: Fundamentos; 1998.

21. Braun V, Clarke V. Using thematic analysis in psychology. Qualitative Research in Psychology 2006; 3(2): 77-101.

Recibido: 17 de julio de 2020

Aceptado: 6 de agosto de 2020 